\title{
Crescimento e mortalidade de Brycon opalinus (Characiformes, Characidae) no Parque Estadual da Serra do Mar, Mata Atlântica, Estado de São Paulo
}

\author{
Leandro Muller Gomiero ${ }^{1,2}$, Alberto Luciano Carmassi ${ }^{1}$ \& Francisco Manoel de Souza Braga $^{1}$
}

Biota Neotropica v7 (n1)-http://www.biotaneotropica.org.br/v7n1/pt/abstract?article+bn00207012007

Recebido em 21/07/06

Versão reformulada recebida 16/10/06

Publicado em 01/01/07

${ }^{1}$ Departamento de Zoologia, Instituto de Biociências, Universidade Estadual Paulista - UNESP,

CP 199, CEP 13506-900, Av. 24-A, n. 1515, Rio Claro, São Paulo, Brasil

${ }^{2}$ Autor para correspondência: Leandro Muller Gomiero, e-mail: leanmg@rc.unesp.br

\section{Abstract}

Gomiero, L.M., Carmassi, A.L. \& Braga, F.M.S. Growth and mortality of Brycon opalinus (Characiformes, Characidae) in Serra do Mar State Park, Atlantic Forest, São Paulo State. Biota Neotrop. Jan/Apr 2007 vol. 7 , no. 1 http://www.biotaneotropica.org.br/v7n1/pt/abstract?article+bn00207012007 ISSN 1676-0603.

Growth and mortality parameters for males and females of Brycon opalinus were estimated from January through December 2004 in rivers of the Serra do Mar State Park - Santa Virgínia Unit, State of São Paulo. The distributions of the total-length frequency classes were different for males and females. The estimated parameters for males and females were: $\mathrm{L}_{\infty}$ (asymptotic maximum length) $=29.8$ and $37.7 \mathrm{~cm}$; $\mathrm{k}$ (growth coefficient $)=0.54$ and 0.56 year $^{-1} ; \mathrm{C}$ (growth oscillation $)=0.3 ; \mathrm{Wp}$ (winter point $)=0.7 ; \mathrm{M}$ (natural mortality coefficient $)=0.97$ and 0.93 year $^{-1}$; four to five cohorts and longevity of 4.58 and 4.53 years, respectively. Growth and mortality of $B$. opalinus are intimately related to the intrinsic characteristics of the species, as well as to the environmental characteristics of seasonality and availability of food.

Keywords: Pirapitinga do Sul, Santa Virgínia Unit, Brazil.

\section{Resumo}

Gomiero, L.M., Carmassi, A.L. \& Braga, F.M.S. Crescimento e mortalidade de Brycon opalinus (Characiformes, Characidae) no Parque Estadual da Serra do Mar, Mata Atlântica, estado de São Paulo. Biota Neotrop. Jan/Apr 2007 vol. 7, no. 1 http://www.biotaneotropica.org.br/v7n1/pt/abstract?article+bn00207012007 ISSN 1676-0603.

Os parâmetros de crescimento e mortalidade de machos e fêmeas de Brycon opalinus foram estimados de janeiro a dezembro de 2004 em rios do Parque Estadual da Serra do Mar - Núcleo Santa Virgínia (SP). As distribuições das frequiências nas classes de comprimentos totais foram diferentes para machos e fêmeas. Os parâmetros estimados para os machos e fêmeas foram: Lo (comprimento médio máximo assintótico) $=29,8$ e $37,7 \mathrm{~cm} ; \mathrm{k}$ (coeficiente de taxa de crescimento) $=0,54$ e 0,56 ano $^{-1} ; \mathrm{C}$ (amplitude de oscilação sazonal $)=0,3$; $\mathrm{Wp}$ ("winter point") $=0,7 ; \mathrm{M}$ (coeficiente de mortalidade natural) $=0,97$ e 0,93 ano $^{-1}$; quatro a cinco coortes e longevidade de 4,58 e 4,53 anos, respectivamente. O crescimento e a mortalidade de B. opalinus estão intimamente relacionados com as características intrínsecas da espécie, assim como com as características ambientais de sazonalidade e de disponibilidade alimentar.

Palavras-chave: Pirapitinga do Sul, Núcleo Santa Virgínia, Brasil. 


\section{Introdução}

O mais importante atributo de um organismo é o seu tamanho, o qual determina grandemente a natureza de suas interações com outros organismos e suas características demográficas (Pauly 1998). Para estimar a taxa de crescimento é necessário determinar os grupos etários e taxas de crescimento da população, o que não é tarefa fácil, especialmente em peixes sob condições tropicais, pois as marcas que definem os anéis de crescimento são pouco visíveis (Lowe-McConnell 1999).

As escamas são freqüentemente utilizadas nos estudos de crescimento de peixes, porém a determinação da idade através das escamas também é difícil em regiões tropicais (Boujard et al. 1991) e a validação das classes etárias nem sempre é possível (Lizama \& Vazzoler 1993). Assim, em estudos com animais em ambiente natural os parâmetros de crescimento são geralmente estimados indiretamente (Araújo-Lima \& Goulding 1998), por meio de dados de freqüência de comprimento, que contêm informações sobre o comprimento médio em cada grupo etário, e pela posição das modas associadas com as coortes (Basson et al. 1988).

Nas altas temperaturas prevalecentes em águas tropicais, as taxas de crescimento são mais rápidas, o peixe matura com menor idade e o período de vida é mais curto do que em águas temperadas (LoweMcConnell 1999). Acredita-se que em regiões temperadas a taxa de crescimento dos peixes seja afetada principalmente pela temperatura, enquanto que em regiões tropicais os recursos alimentares atuem como fatores restritivos ao crescimento (Boujard et al. 1991).

Em estudos de dinâmica de populações de peixes, nota-se que o crescimento e a mortalidade interagem de tal forma que, se o crescimento contribui para a abundância de espécies, fazendo com que ela atinja a fase de reprodução em um dado momento e entre no ciclo reprodutivo, a mortalidade controla a abundância dessa espécie (Nikolskii 1969). Dessa forma, as informações sobre o crescimento e a maturidade são essenciais para o manejo das populações de peixes (Froese \& Binohlan 2000), além de contribuírem para o conhecimento da dinâmica e ecologia populacional das espécies, especialmente aquelas ameaçadas de extinção.

A pirapitinga do sul, Brycon opalinus (Cuvier 1819), encontra-se ameaçada de extinção (Brasil 2003, 2004), sendo um peixe de rios e riachos de encosta da bacia do rio Paraíba do Sul (Lima 2003), em áreas com remanescentes de floresta ripária em boas condições de preservação. Esta bacia localiza-se no extremo norte da zona da Floresta Ombrófila Densa do Estado de São Paulo e de modo geral a região possui alta percentagem de espécies endêmicas de peixes (Buckup 2003). No presente estudo, determinamos os parâmetros de crescimento e mortalidade de machos e fêmeas de B. opalinus capturados no Parque Estadual da Serra do Mar, Núcleo Santa Virgínia.

\section{Material e Métodos}

Os exemplares foram capturados em três localidades da bacia do Rio Paraibuna, no Núcleo Santa Virgínia do Parque Estadual da Serra do Mar $\left(23^{\circ} 24^{\prime}-23^{\circ} 17^{\prime} \mathrm{S}\right.$ e $\left.45^{\circ} 03^{\prime} \mathrm{W}\right)$ : rios Paraibuna, Ipiranga e Grande.

Foram efetuadas 12 amostragens mensais de janeiro a dezembro de 2004. Para a captura dos exemplares foram utilizados conjuntos de redes de espera (malhas de 1,$5 ; 2,0 ; 2,5 ; 3,0 ; 3,5$ e $4 \mathrm{~cm}$ ) com $10 \mathrm{~m}$ de comprimento e $1,5 \mathrm{~m}$ de altura, totalizando $60 \mathrm{~m}$. O esforço de pesca foi padronizado mantendo-se constante o tempo e o número dos apetrechos de pesca utilizados em cada local de amostragem. Para cada exemplar foram obtidos os dados de comprimento total $(\mathrm{cm})$ e sexo (Braga 1990). Os exemplares foram fixados, conservados e depositados no Departamento de Zoologia da Universidade Estadual Paulista (UNESP) em Rio Claro, São Paulo.
A curva de crescimento foi obtida por meio do modelo de von Bertalanffy, dado por: $\mathrm{L}_{\mathrm{t}}=\mathrm{L} \infty\left(1-\mathrm{e}^{-\mathrm{k}(\mathrm{t}-\mathrm{to})}\right)$, onde: $\mathrm{L}_{\mathrm{t}}=$ comprimento na idade $\mathrm{t} ; \mathrm{L} \infty=$ comprimento médio máximo assintótico ou máximo teórico que a espécie pode atingir; $\mathrm{k}=$ coeficiente de taxa de crescimento, que determina quão rapidamente o máximo é atingido; e $\mathrm{t}_{0}=$ idade teórica no comprimento zero.

Os parâmetros de crescimento foram estimados com o método ELEFAN I (Eletronic Lengths-Frequency Analysis) (Pauly \& David 1981), inserido no pacote computacional FISAT (FAO-ICLARM Stock Assessment Tools) (Gayanilo et al. 1996, Gayanilo \& Pauly 1997) e que se baseia no deslocamento modal de seqüências temporais de amostras de comprimento (Castro et al. 2002), utilizando-se a versão sazonal da curva de crescimento de von Bertalanffy (Sparre \& Venema 1992), que considera, além dos parâmetros L $\infty$ e k, outros dois parâmetros: Wp ("winter point") e C (amplitude de oscilação sazonal). O valor de $\mathrm{C}$ está relacionado à diferença de temperaturas superficiais médias da água de inverno e verão a que o peixe está submetido; $\mathrm{Wp}$ representa a época do ano em que o crescimento é mais lento, sendo uma relação do número de dias até o mês de menor crescimento da espécie com o número de dias do ano (Castro et al. 2002).

O método de Bhattacharya (1967 apud Sparre \& Venema 1992) foi utilizado para separar em distribuições normais a distribuição composta obtida para cada sexo. Este método consiste basicamente na separação destas distribuições, sendo que cada uma representa uma coorte de peixes da distribuição global.

O comprimento assintótico $(\mathrm{L} \infty)$ foi estimado a partir do maior indivíduo capturado $\left(\mathrm{L}_{\text {máx }}\right)$, sendo: $\mathrm{L}_{\infty}=\mathrm{L}_{\text {máx }} / 0,95$ (Pauly, 1983).

$O$ índice de performance de crescimento $\left(\varphi^{\prime}\right)$ foi utilizado para comparar as curvas de crescimento de machos e de fêmeas de B. opalinus, segundo a equação de Pauly \& Munro (1984 apud Castro et al. 2002): $\varphi^{\prime}=\log \mathrm{k}+2 \log \mathrm{L} \infty$.

Como não é provável que uma espécie de peixe cresça seguindo essa equação desde o instante em que nasce até atingir a senilidade, a curva freqüientemente corta o eixo $\mathrm{x}$, referente à idade, em um ponto geralmente menor do que zero (King 1996). Para o cálculo do parâmetro $t_{0}$, idade teórica no comprimento zero, da equação de crescimento de von Bertalanffy, utilizou-se a fórmula empírica de Pauly (1979): $\log \left(-t_{0}\right)=-0,3922-0,2752 \log L \infty-1,038 \log k$.

A longevidade, definida como o tempo que o indivíduo leva para alcançar 95\% do comprimento assintótico, foi estimada com base na fórmula proposta por Taylor (1958): $\mathrm{t}_{\text {máx }}=\mathrm{t}_{0}+2,996 / \mathrm{k}$.

O coeficiente de mortalidade natural (M) foi obtido empregando-se a fórmula empírica proposta por Pauly (1980), que requer os parâmetros de crescimento (Lo e k) e a temperatura média $\left(\mathrm{T}^{\circ}\right)$ do ambiente em que a espécie se insere: $\ln \mathrm{M}=-0,0152-0,279 \ln$ $\mathrm{L} \infty+0,6543 \ln \mathrm{k}+0,463 \ln \mathrm{T}^{\circ}$.

\section{Resultados}

Foram utilizados os dados de comprimento total de 77 machos e de 171 fêmeas de B. opalinus. Os machos variaram de 17,2 a 28,3 cm e as fêmeas de 17,8 a $35,8 \mathrm{~cm}$. As distribuições de comprimento total para machos e fêmeas em todo período mostram que as fêmeas sempre foram mais abundantes nas classes de comprimentos mais elevadas, já os machos foram abundantes nas classes inferiores (Figura 1).

As distribuições de classes de comprimento total de machos e de fêmeas de B. opalinus em cada estação do ano mostram que houve variações ao longo do período das coletas. Estas distribuições sempre foram polimodais, sendo que os machos apresentaram no inverno uma menor abundância de indivíduos. Na primavera houve maior frequiência nas classes de comprimentos mais elevadas, já no verão a maior freqüência ocorreu nas menores classes. Houve um deslocamento das freqüências de ocorrências das classes de comprimentos inferiores 
para as mais elevadas do outono para o inverno (Figura 2). As fêmeas foram mais freqüentes nas maiores classes de comprimentos no outono e na primavera, sendo observado o deslocamento da frequiência para as maiores modas de comprimentos, principalmente, do inverno para a primavera e verão e deste para o outono (Figura 3).

As curvas de crescimento e as coortes obtidas para machos e fêmeas de B. opalinus pelo método ELEFAN I estão na Figura 4. Cinco coortes foram registradas, sendo que para os machos cada coorte surge no outono e para as fêmeas no verão. A desova desta espécie é do tipo total com dois picos, um na primavera-verão e outro no início do outono (L.M. Gomiero \& F.M.S.Braga, dados não publicados). Pelo método de Bhattacharya constatou-se a presença de quatro coortes tanto para os machos quanto para as fêmeas.

A amplitude de oscilação sazonal foi de 0,3 , correspondendo assim, a uma diferença de $3{ }^{\circ} \mathrm{C}$ entre as temperaturas superficiais médias da água entre o inverno e o verão, sendo que o Wp ("winter

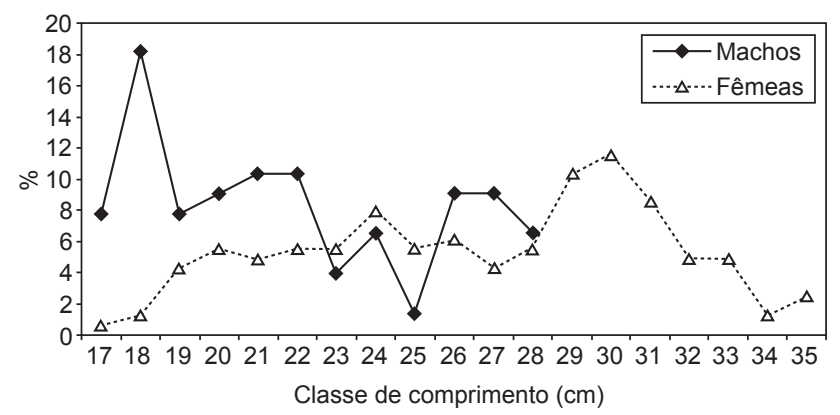

Figura 1. Distribuição porcentual nas classes de comprimento total de machos e fêmeas de Brycon opalinus.

Figure 1. Percent distribution in the total length classes of male and female Brycon opalinus.
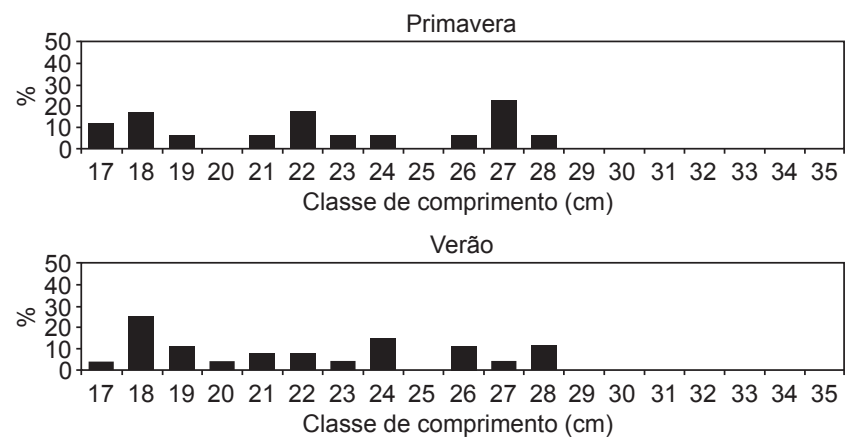

Outono
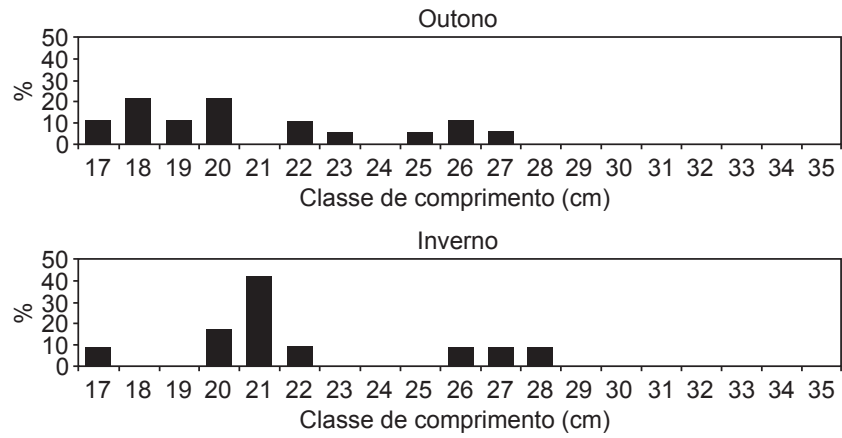

Figura 2. Distribuição porcentual nas classes de comprimento total de machos de Brycon opalinus em cada estação do ano.

Figure 2. Percent distribution in the total length classes of male Brycon opalinus in each season of the year. point") foi de 0,7 , o que equivale a agosto/setembro, representando a época do ano em que o crescimento é mais lento. Os valores do índice de performance de crescimento $(\varphi$ ') entre machos e fêmeas foram muito próximos, mostrando que o conjunto de parâmetros estimados
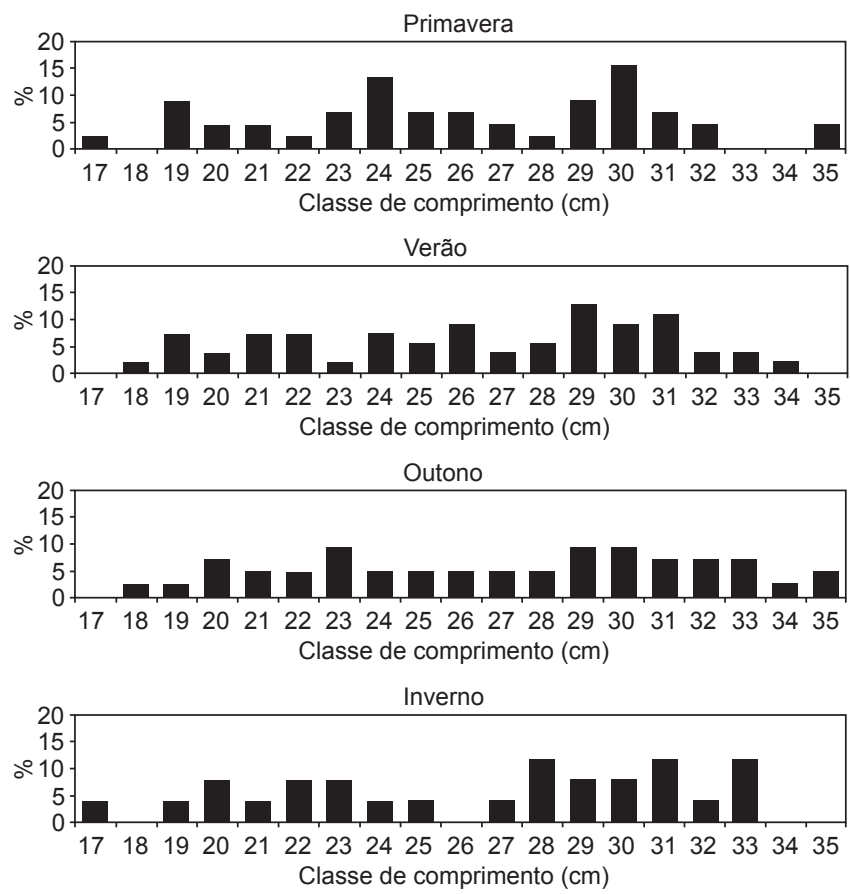

Figura 3. Distribuição porcentual nas classes de comprimento total de fêmeas de Brycon opalinus em cada estação do ano.

Figure 3. Percent distribution in the total length classes of female Brycon opalinus in each season of the year.
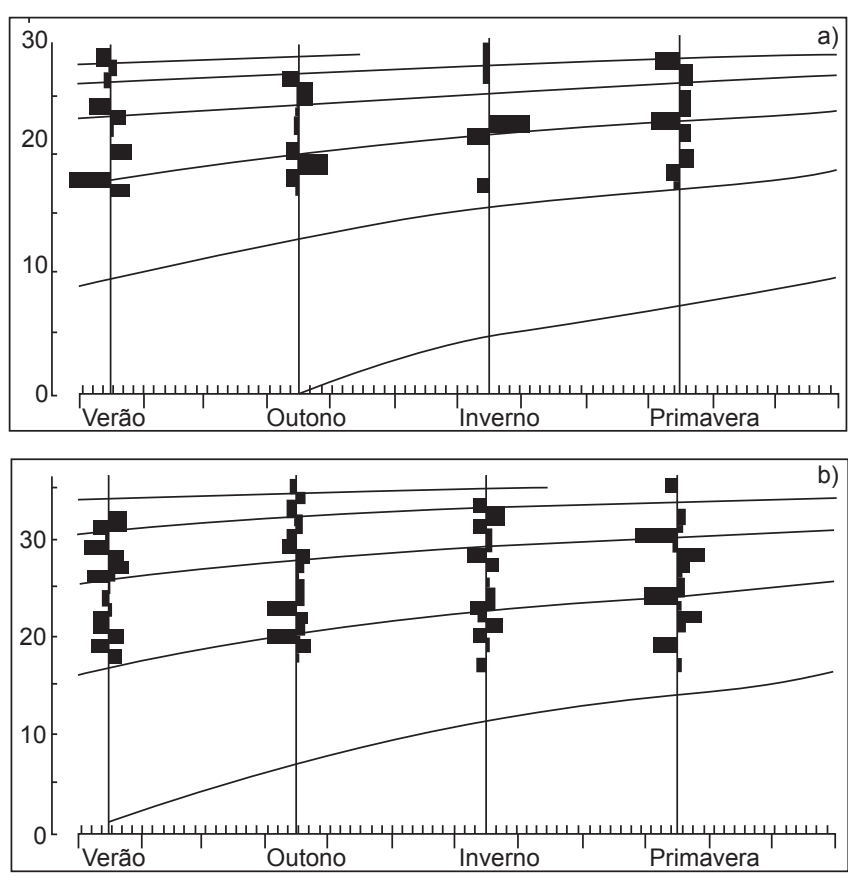

Figura 4. Curvas de crescimento das coortes de machos a) e fêmeas b) de Brycon opalinus, calculadas por meio do sistema ELEFAN I (pacote computacional FISAT).

Figure 4. Growth curves of Brycon opalinus males a) and females b) cohorts, calculated by the ELEFAN 1 system (FISAT computational package). 
diferem pouco entre si. A taxa instantânea de mortalidade total $(Z)$ foi a mesma que a taxa instantânea de mortalidade natural (M), devido a não exploração do seu estoque (Pauly 1998). A mortalidade natural foi elevada tanto para os machos $(0,97)$ quanto para as fêmeas $(0,93)$ e a longevidade de machos e de fêmeas foi praticamente a mesma, estimada em 4 anos e meio.

Os parâmetros de crescimento, de mortalidade e as melhores combinações obtidas pelo ELEFAN I, em função dos melhores ajustes ou maiores valores de Rn ("goodness of fit index") para Brycon microlepis (piraputanga) e Brycon cephalus (matrinxã) foram apresentados para comparações com os parâmetros obtidos de Brycon opalinus (Tabela 1).

\section{Discussão}

O modelo mais utilizado para descrever a curva de crescimento em peixes é o de von Bertalanffy (Penna et al. 2005). Quando as estruturas calcificadas dos peixes não estão disponíveis ou a leitura dos anéis de crescimento é confusa, sugere-se o método ELEFAN I como uma boa alternativa para a determinação dos parâmetros de crescimento, levando-se em conta a distribuição temporal das modas de freqüência de comprimento.

Machos e fêmeas de $B$. opalinus têm taxas de crescimento similares, que foram muito próximas àquelas encontradas para $B$. cephalus (matrinxã) na Amazônia, porém superiores às de B. microlepis (piraputanga) no Pantanal. Esta espécie apresentou o comprimento máximo e o número de coortes muito superiores aos de B. opalinus, sendo, portanto muito maior e mais longeva do que $B$. opalinus e B. cephalus (Tabela 1).

Há uma relação inversa entre k (taxa de crescimento) e o Lo (comprimento assintótico), sendo que quanto maior a taxa de crescimento, menor será o comprimento assintótico e o período de vida (Beverton \& Holt 1957, Ricker 1975). O comprimento assintótico é afetado por fatores como suprimento alimentar e de densidade populacional enquanto que a taxa de crescimento é determinada genética e/ou fisiologicamente (Beverton \& Holt 1957).
O crescimento de $B$. microlepis segue um padrão oscilatório sazonal com uma taxa de crescimento lenta entre maio e junho correspondendo ao regime de águas baixas do Pantanal (Mateus \& Estupiñán 2002). Para B. opalinus, o padrão de crescimento parece ser o mesmo, com a taxa de crescimento lenta entre agosto e setembro, coincidindo com a época em que as reservas de gordura já foram gastas (inverno) e ainda ocorre escassez de recursos alimentares. O principal fator afetando o crescimento é a extensão do período chuvoso que corresponde ao período de maior atividade alimentar (Boujard et al. 1991). Contudo, em regiões de Floresta Ombrófila (local das amostragens), as condições climáticas são pouco variáveis e a umidade é relativamente elevada o ano todo, como resultado de influências oceânicas (Tonhasca Jr. 2005), indicando que outras variáveis, tais como temperatura relativamente baixa da água, disponibilidade de insetos e/ou fenologia das espécies arbóreas que fornecem itens alimentares para B. opalinus, podem estar envolvidas no crescimento dessa espécie.

O crescimento dos peixes é diretamente relacionado com a massa da população, que é controlada pela reprodução e influenciada pelo suprimento alimentar (Nikolskii 1969). Ainda segundo este autor, a principal fração de proteína alimentar é convertida em crescimento e conseqüiente aumento do comprimento antes da maturidade, posteriormente o alimento é utilizado principalmente para o aumento do peso e das reservas para a migração reprodutiva e desenvolvimento das gônadas.

A alimentação dos peixes do gênero Brycon consiste principalmente de material vegetal (folhas, flores, frutos e sementes) e de material animal (insetos terrestres e ocasionalmente peixes). Esta dieta pode variar ontogeneticamente, tornando-se cada vez mais herbívora com o crescimento dos peixes (Menezes 1969, Correa 1981). De acordo com Grimes (1978), quanto menor o nível trófico do alimento de uma espécie, maior será sua taxa de crescimento, o que significa que quanto menor o nível trófico do item alimentar, maior a energia acumulada (primeiros níveis tróficos da pirâmide de energia), fornecendo maior suprimento energético para o peixe com menor custo de captura e digestão do alimento (Lizama \& Vazzoler 1993). Para o tambaqui, Colossoma macropomum, o crescimento é influenciado

Tabela 1. Parâmetros de crescimento, mortalidade, comprimento máximo $\left(\mathrm{L}_{\text {max }}\right)$ e mínimo $\left(\mathrm{L}_{\text {min }}\right)$ dos indivíduos capturados, temperatura média $\left(\mathrm{T}^{\circ}\right)$ e a longevidade para Brycon microlepis (Pantanal), Brycon cephalus (Amazônia) e Brycon opalinus (Mata Atlântica). "nd" indica informações não disponíveis.

Table 1. Parameters of growth, mortality, maximum $\left(\mathrm{L}_{\max }\right)$ and minimum $\left(\mathrm{L}_{\min }\right)$ length of captured individuals, mean temperature $\left(\mathrm{T}^{\circ}\right)$ and longevity of $B r y c o n$ microlepis (Pantanal), Brycon cephalus (Amazonia) and Brycon opalinus (Atlantic Forest). "nd" indicates not available information.

\begin{tabular}{|c|c|c|c|}
\hline Parâmetros & $\begin{array}{c}\text { B. microlepis } \\
\text { (Mateus \& Estupiñán 2002) } \\
1996-1997\end{array}$ & $\begin{array}{c}\text { B. cephalus } \\
\text { (Villacorta-Correa 1987) } \\
\text { Machos - Fêmeas } \\
\end{array}$ & $\begin{array}{c}\text { B. opalinus } \\
\text { (presente estudo) } \\
\text { Machos - Fêmeas } \\
\end{array}$ \\
\hline $\mathrm{L} \infty(\mathrm{cm})$ & $70-71$ & $41,6-44,9$ & $29,8-37,7$ \\
\hline $\mathrm{k}\left(\mathrm{ano}^{-1}\right)$ & $0,28-0,27$ & $0,68-0,54$ & $0,545-0,560$ \\
\hline $\mathrm{t}_{0}$ & nd & $(-0,70)-(-0,84)$ & $(-0,498)-(-0,454)$ \\
\hline $\mathrm{C}$ & $0,75-0,8$ & nd & 0,3 \\
\hline Wp & $0,5-0,43$ & nd & 0,7 \\
\hline$\varphi^{\prime}$ & nd & nd & $6,18-6,67$ \\
\hline $\mathrm{Rn}$ & $0,174-0,154$ & nd & $0,242-0,155$ \\
\hline $\mathrm{Z}\left(\mathrm{ano}^{-1}\right)$ & $0,831-0,814$ & nd & $0,977-0,932$ \\
\hline $\mathrm{M}\left(\mathrm{ano}^{-1}\right)$ & $0,59-0,58$ & nd & $0,977-0,932$ \\
\hline $\mathrm{L}_{\text {máx }}(\mathrm{cm})$ & $67-59$ & 45,9 & $28,3-35,8$ \\
\hline $\mathrm{L}_{\operatorname{mín}}(\mathrm{cm})$ & $16,4-20$ & nd & $17,2-17,8$ \\
\hline $\mathrm{T}^{\circ}\left({ }^{\circ} \mathrm{C}\right)$ & nd & nd & $17,9-17,9$ \\
\hline $\mathrm{N}^{\mathrm{o}}$ de coortes & $10-7$ & nd & $5-5$ \\
\hline Longevidade (ano) & nd & nd & $4,58-4,53$ \\
\hline
\end{tabular}


pela temperatura, quantidade e qualidade dos itens alimentares nas planícies inundadas Amazônicas (Araújo-Lima \& Goulding 1998).

Em muitas espécies de peixes as taxas de crescimento variam acentuadamente de acordo com as condições ambientais, a disponibilidade de alimento, e fatores que afetam sua utilização como a temperatura e o adensamento populacional. Estas taxas também podem variar durante o ciclo de vida, sazonalmente e em cada hábitat ocupado pela espécie (Lowe-McConnell 1999). Ao longo do ciclo vital, os indivíduos de uma classe etária ficam sujeitos a níveis e elementos predatórios diversos, em que a mortalidade natural se irá reduzindo à medida que aumentam de tamanho, tornando-se vulneráveis a um número cada vez menor de predadores (Fonteles-Filho 1989).

As diferentes espécies de peixes tendem a ter taxas de mortalidade altas ou baixas, que são adaptações ao ambiente em que vivem, e que interferem na biomassa dessas populações. Brycon opalinus é uma espécie que habita riachos de montanha, tendo um ecoespaço limitado. Por outro lado, a sua desova é do tipo demersal sem cuidado parental (JP. Villani, com. pess.), sendo os ovos depositados no sedimento e as larvas sujeitas a dispersão e a diferentes tipos de predação, fazendo com que esta espécie tenha uma fecundidade populacional elevada (LM. Gomiero \& FMS. Braga, dados não publicados), para compensar a alta taxa de mortalidade.

Os peixes tropicais tendem a ter, para qualquer tamanho assintótico ou de taxa de crescimento, altos valores de mortalidade natural (Pauly 1998). Os principais fatores naturais de mortalidade são a predação e as doenças, que apresentam intensidade de ação com tendências divergentes em função da idade, isto é, indivíduos maiores e mais velhos sofrem menor mortalidade por predação, mas são mais suscetíveis a doenças devido à degeneração dos tecidos e menor aproveitamento dos elementos nutritivos (Fonteles-Filho 1989).

As taxas de mortalidade e os parâmetros da curva de crescimento de von Bertalanffy são com frequiência utilizados em modelos de monitoramento de estoques de peixes (Braga 2001), tendo por isso função importantíssima para planos de manejo de uma espécie ameaçada de extinção como $B$. opalinus. O crescimento e a mortalidade de $B$. opalinus estão intimamente relacionados com as características intrínsecas da espécie, assim como com as características ambientais de sazonalidade e de disponibilidade alimentar.

\section{Agradecimentos}

À FAPESP (03/05696-1) pela bolsa de pós-doutorado, à Cotec (40.673/03), ao IBAMA (055/2003) e ao Sr. J. P. Villani (Diretor do PESM-Núcleo Santa Virgínia) por proporcionar as condições necessárias para o desenvolvimento desta pesquisa.

\section{Referências Bibliográficas}

ARAÚJO-LIMA, C. \& GOULDING, M. 1998. Os frutos do Tambaqui. Ecologia, conservação e cultivo na Amazônia. MCT-CNPq, Tefé-AM.

BASSON, M., ROSENBERG, A.A. \& BEDDINGTON, J.R. 1988. The accuracy and reliability of two new methods for estimating growth parameters from length-frequency data. J. Cons. Int. Explor. Mer. 44:277-285.

BEVERTON, R.J.H. \& HOLT, S.J. 1957. On the dynamics of exploited fish populations. Fishery Invest. London. 2(19):1-533.

BOUJARD, T., LECOMTE, F., RENNO, J.F., MEUNIER, F. \& NEVEU, P. 1991. Growth in four populations of Leporinus friderici (Bloch, 1794) (Anostomidae, Teleostei) in French Guiana. J. Fish Biol. 38:387-397.

BRAGA, F.M.S. 1990. Aspectos da reprodução e alimentação de peixes comuns em um trecho do rio Tocantins entre Imperatriz e Estreito, Estados do Maranhão e Tocantins, Brasil. Rev. Bras. Biol. 50(3):547-558.
BRAGA, F.M.S. 2001. Crescimento e mortalidade de Leporinus friderici (Ostariophysi, Anostomidae) na represa de Volta Grande, rio Grande, localizada entre os Estados de Minas Gerais e São Paulo, Brasil. Acta Scientiarum 23(2):415-420.

BRASIL, 2003. Instrução Normativa n³, de 27 de maio de 2003. Reconhece espécies da fauna brasileira ameaçadas de extinção. Ministério do Meio Ambiente, Brasília.

BRASIL, 2004. Instrução Normativa n5, de 21 de maio de 2004. Reconhece espécies de invertebrados aquáticos e peixes ameaçados de extinção, sobreexplotadas ou ameaçadas de sobreexplotação. Ministério do Meio Ambiente, Brasília.

BUCKUP, P.A. 2003. Biodiversidade dos peixes da Mata Atlântica. Disponível em: <http://www.bdt.fat.org.br/workshop/mata.atlantica/SE-S/peixes. Acessado em 18.mar.2003.

CASTRO, P.M.G., CERGOLE, M.C., CARNEIRO, M.H., MUCINHATO, C.M.D. \& SERVO, G.J.M. 2002. Crescimento, mortalidade e taxa de explotação do Goete, Cynoscion jamaicensis (Perciformes: Scianidae), na região Sudeste/Sul do Brasil. Bol. Inst. Pesca 28(2):141-153.

CORRÊA, V.L.S. 1981. Considerações sobre o hábito alimentar do Brycon devillei. An. Acad. Brasil. Ciênc. 53(3):630-631.

FONTELES-FILHO, A.A. 1989. Recursos pesqueiros. Biologia e dinâmica populacional. Ed. da Imprensa Oficial do Ceará, Fortaleza.

FROESE, R. \& BINOHLAN, C. 2000. Empirical relationships to estimate asymptotic length, length at first maturity and length at maximum yield per recruit frequency data. J. Fish Biol. 56:758-773.

GAYANILO JR., F.C. \& PAULY, D. 1997. FAO-ICLARM Stock Assessment Tools (FISAT) Reference manual. FAO Computerized Information Series (Fisheries) 8:1-262.

GAYANILO JR., F.C., SPARRE, P. \& PAULY, D. 1996. The FAO-ICLARM Stock Assessment Tools (FISAT) User's guide. FAO Computerized Information Series (Fisheries) 6:1-186.

GRIMES, C.B. 1978. Growth and length-weight relationship of vermilion snapper, Rhomboplites aurorubens, from North Carolina and South Carolina waters. Trans. Am. Fish. Soc. 107:454-456.

KING, M. 1996. Fisheries biology, assessment and management. Ed. Blackwell Science. Oxford.

LIMA, F.C.T. 2003. Subfamily Bryconinae (Characins, Tetras). In Check List of the Freshwater Fishes of South and Central America (CLOFFSCA) (R.E. Reis, S.O. Kullander \& C.J. Ferraris Jr., orgs.). EDIPUCRS, Porto Alegre, p.174-181.

LIZAMA, M.L.A.P. \& VAZZOLER, A.E.A.M. 1993. Crescimento em peixes no Brasil: uma síntese comentada. Rev. UNIMAR. 15:141-173.

LOWE-McCONNELL, R.H. 1999. Estudos ecológicos de comunidades de peixes tropicais. Ed. da Universidade de São Paulo, São Paulo.

MATEUS, L.A. \& ESTUPIÑÁN, G.M.B. 2002. Fish stock assessment of piraputanga Brycon microlepis in the Cuiabá river basin, Pantanal of Mato Grosso, Brazil. Braz. J. Biol. 62(1):165-170.

MENEZES, N.A. 1969. The food of Brycon and three closely related genera of the tribe Acestrorhynchini. Pap. Av. Zool. 22:217-223.

NIKOLSKII, G.V. 1969. Theory of fish population dynamics. Ed. Oliver \& Boyd. Edinburgh.

PAULY, D. 1979. Theory and management of tropical multispecies stocks-A review, with emphasis on the southeast Asia demersal fisheries. ICLARM Studies \& Review. 1:1-35.

PAULY, D. 1980. On the interrelationships between natural mortality, growth parameters and mean environmental temperature in 175 fish stock. International Council for the Exploration of the Sea 39:175-192.

PAULY, D. 1983. Some simple methods for the assessment of tropical fish stocks. FAO Fisheries Technical Paper 234:1-52.

PAULY, D. 1998. Tropical fishes: patterns and propensities. J. Fish Biol. 53:1-17. 
PAULY, D. \& DAVID, N. 1981. ELEFAN I, a BASIC program for the objective extraction of growth parameters from length-frequency data. Meeresforschung. 28(4):205-211.

PENNA, M.A.H., VILLACORTA-CORRÊA, M.A., WALTER, T. \& PETRERE-JR., M. 2005. Growth of the Tambaqui Colossoma macropomum (Cuvier) (Characiformes: Characidae): Which is the best model? Braz. J. Biol. 65(1):129-139.

RICKER, W.E. 1975. Computation and interpretation of biological statistics of fish populations. Bull. Fish. Res. Board. Can. 191:1-382.

SPARRE, P. \& VENEMA, S.C. 1992. Introduction to tropical fish stock assessment. Part 1. FAO Fisheries Technical Paper 306:1-376.

TAYLOR, C.C. 1958. Cod growth and temperature. J. Cons. Int. Explor. Mer. 23:366-370.

TONHASCA JR., A. 2005. Ecologia e história natural da Mata Atlântica. Ed. Interciência.

VILLACORTA-CORREA, M.A. 1987. Crescimento do matrinxã, Brycon cephalus (Günther, 1869) (Teleostei, characidae) no rio Negro, seus afluentes e no baixo rio Solimões. Dissertação de Mestrado, Instituto Nacional de Pesquisas da Amazônia/Fundação Universidade do Amazonas, Manaus.
Título: Crescimento e mortalidade de Brycon opalinus (Characiformes, Characidae) no Parque Estadual da Serra do Mar, Mata Atlântica, Estado de São Paulo.

Autores: Gomiero, LM, Carmassi, AL e Braga, FMS

Biota Neotropica, Vol.7 (número 1): 2007

http://www.biotaneotropica.org.br/v7n1/pt/abstract?article+bn 00207012007

Recebido em 21/07/06 - Versão reformulada recebida em 16/10/06 - Publicado em 01/01/07

ISSN 1676-0603 